J. Indones. Math. Soc. Special Edition (2011), pp. 71-78.

\title{
ON THE EDGE-BALANCED INDEX SETS OF PRODUCT GRAPHS
}

\author{
Elliot Krop ${ }^{1}$, Sin-Min LeE ${ }^{2}$, Christopher Raridan ${ }^{3}$ \\ ${ }^{1}$ Department of Mathematics, Clayton State University, Morrow, GA 30260 , \\ USA, ElliotKrop@clayton.edu \\ ${ }^{2}$ Department of Computer Science, San Jose State University, San Jose, CA \\ 95192, lee.sinmin35@gmail.com \\ ${ }^{3}$ Department of Mathematics, Clayton State University, Morrow, GA 30260, \\ USA, ChristopherRaridan@clayton.edu
}

\begin{abstract}
We characterize strongly edge regular product graphs and find the edgebalanced index sets of complete bipartite graphs without a perfect matching, the direct product $K_{n} \times K_{2}$. We also prove a lemma that is helpful to determine the edge-balanced index sets of regular graphs.

Key words: Edge-labeling, partial-coloring, edge-friendly labeling, friendly labeling, cordiality, friendly index set, edge-balanced index set.
\end{abstract}

2000 Mathematics Subject Classification: 05C78, 05C25.

Received: 09-08-2011, revised: 09-09-2011, accepted: 04-12-2012. 